\title{
Simple model of the rf noise generated by multipacting electrons.
}

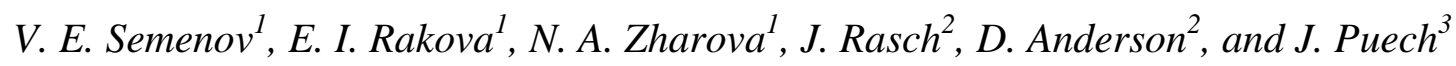 \\ 1) Institute of Applied Physics, Nizhny Novgorod, Russia \\ 2) Chalmers University of Technology, Göteborg, Sweden \\ 3) CNES, Toulouse, France
}

\begin{abstract}
A simplified analytical model is developed to predict the spectrum of electric current induced by the multipacting electrons between two parallel electrodes exposed to an rf voltage of fixed amplitude. The model is based on the resonant multipactor theory and makes it possible to calculate the relative spectral amplitudes of electric current at different harmonics and sub-harmonics of the applied rf frequency. The theoretical predictions are confirmed by numerical simulations of multipactor inside a rectangular waveguide. Specifically it is seen that the relative height of the spectral peaks decreases with increasing gap height.
\end{abstract}

\section{Introduction}

The multipactor phenomenon is usually associated with the generation of electromagnetic noise in $\mathrm{rf}$ components. The noise intensity increases rapidly with an increase in the transmitted rf power and therefore this noise is often considered as an indication of a multipactor discharge occurring in the component [1]. It is commonly accepted to distinguish the close-to-carrier noise from the noise around the harmonics of the transmitted signal frequency. Specifically, the noise around the third harmonic is the most well-known and its measurement has been suggested as a means of detecting the appearance of multipactor in various devices [2]. The electromagnetic noise is generated by an electric current which is related to the motion of the multipacting electrons. On average this motion is repeated with the period, $2 \pi / \omega_{0}$, of the input electromagnetic field at the saturation stage of the multipactor

discharge. In this case the electron motion results in a periodic electric current which generates a series of Fourier harmonics of the input signal frequency. However, in the case of multipactor resonances of higher order, $M \geq 3$, or in the case of hybrid multipactor resonances, the period of the electron motion can exceed $2 \pi / \omega_{0}$ which results in the generation of sub-harmonics of the input frequency [2]. For a pure sinusoidal signal, the close-to-carrier noise as well as the noise around any harmonic or sub-harmonic is caused by fluctuations in the secondary emission yield and the electron emission velocity, which give rise to a stochastic modulation of the multipactor current. In the case of a more complicated signal, it is expected that the finite width of the signal spectrum will give rise to a further widening of the spectral lines of the different harmonics. In this paper we shall restrict ourselves to the case of a pure sinusoidal signal.

Two independent problems should be solved to predict the electromagnetic noise generated by multipactor in any device. First, the electric current density which is associated with multipacting electrons has to be calculated at the saturation stage of the multipactor discharge. Second, the output electromagnetic power which is generated by this electric current should be calculated using the proper electromagnetic field solver. It should be noted that the solution of any specific problem depends on the entire rf circuit, which controls not only the radiation power of any given electric current but also governs the multipactor saturation stage. Actually, it is generally recognized that within the plane-parallel model, multipactor saturation is caused mainly by the space charge effect, 
which can change the electron motion considerably (for example a two-surface multipactor can be transformed into a single-surface multipactor [3-7]). However, in cases where the rf circuit contains one or more resonant cavities (which is a typical situation for any rf filter or transformer), multipactor saturation is caused mainly by impedance mismatch which results in a reduction of the rf voltage amplitude down to the multipactor threshold value $[4,8]$. Therefore a theoretical model has to include the entire rf circuit to predict the multipactor electric current inside separate components of this circuit. This means that accurate quantitative predictions of the multipactor electromagnetic noise are impossible within a simple universal model. A particular model must be developed for each particular rf circuit. Examples of simulations for some rf filter configurations can be found in [9-12].

In the case when a rf circuit contains one or more resonant cavities, there may sometimes be present a narrow gap closely approximating parallel plates. In such a case, when multipactor occurs in this gap, the resulting impedance mismatch will decrease the field in the gap and lead to multipactor saturation. For such a circuit it is possible to suggest a quite simple and universal model capable of predicting quantitatively the spectrum of the specific electric current (current per one electron) which is associated with the multipactor. Knowledge of this spectrum makes it possible to predict the relative spectrum of the output noise power for any similar device, using a commercial electromagnetic field solver. In this paper, we present a description of this model, the analytical results derived from it, and the results from numerical simulations. Our main objective is to find how the height of the noise spectral peaks depend on the gap height.

\section{A simplified model to estimate the multipactor noise spectrum}

Below we will adopt the plane-parallel model to study the generation of electric current harmonics in systems where the multipactor saturation is caused by impedance mismatch. The simplified analytical consideration will be based on the model of multipactor resonance (similar to the model given in [13]) whereas numerical simulations will be carried out taking into account the spread of the electron emission velocity and the non-uniform distribution of the electric field amplitude inside a rectangular waveguide. The resonance model assumes all electrons to be localized within a thin sheet oscillating between parallel metal electrodes. In this case the electric current density can be represented as follows [13]

$$
\vec{j}=(-e N v) \cdot \delta(y-\xi) \cdot \vec{y}_{0},
$$

where $\vec{y}_{0}$ denotes the unit vector perpendicular to the metal surfaces, $-e$ the electron charge, $v(t)$ and $\xi(t)$ the instantaneous velocity and position of the electron sheet, $N$ the number of electrons per unit area of the sheet. When the gap width, $b$, is much smaller than the electromagnetic wavelength, the radiation properties of the electron sheet are determined by an integral, $J$, of the current density over the gap:

$$
J \equiv \int\left(\vec{y}_{0} \cdot \vec{j}\right) d y=-e N v,
$$

At the saturation stage, the electron number, $N$, is constant excepting relatively small stochastic fluctuations. Therefore the process of harmonics generation is determined mainly by the temporal evolution of the electron velocity, $v$. Under the considered approximation (i.e. when impedance mismatch is considered to be the main reason for multipactor saturation), one can neglect the space charge effect and use a very simple model for the electron motion which is governed by the following equation:

$$
\frac{d v}{d t}=\frac{e}{m} \cdot E_{0} \cdot \sin \left(\omega_{0} t\right)
$$

where $m$ denotes electron mass, $E_{0}$ the amplitude of the rf electric field between the parallel metal plates, $\omega_{0}$ the field angular frequency. The solution of (3) can be represented as a superposition of 
forced oscillations of electrons in a monochromatic electric field and a free motion with constant velocity:

$$
v=-V_{o s c} \cos \left(\omega_{0} t\right)+V_{f}
$$

where $V_{o s c}=\frac{e E_{0}}{m \omega_{0}}$ is the amplitude of the electron velocity oscillations, $V_{f}=V_{e}+V_{o s c} \cos \left(\omega_{0} t_{e}\right)$ is the velocity of free electron motion that is determined for each separate electron by the emission time, $t_{e}$, and the emission velocity, $V_{e}$. The forced electron oscillations (the first term on the RHS of (4)) contributes only to the first harmonic of the effective electric current, $J$. Therefore the non-harmonic behavior of the current is determined completely by the free component of the electron velocity, $V_{f}$, which changes in a step-like way after each collision of the electron with the electrodes (see Figs. 1-2).
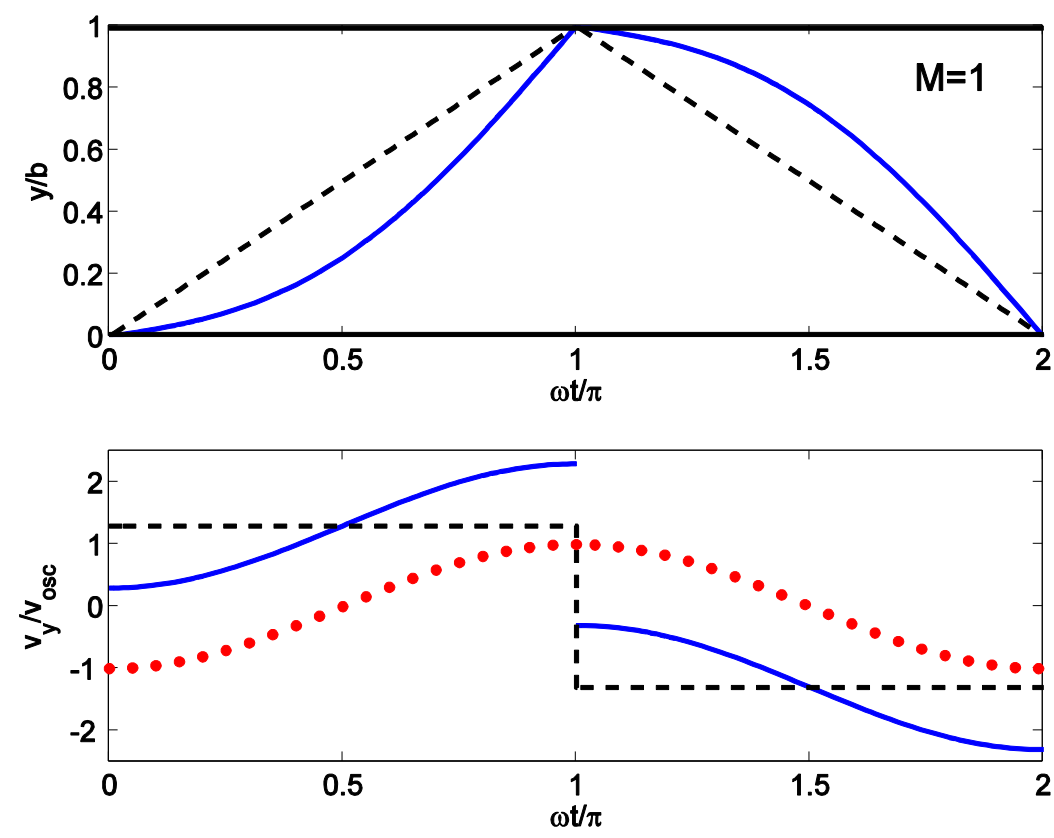

Fig. 1. Electron trajectory (solid blue line on the top panel) and electron velocity (solid blue line on the bottom panel) calculated in the case of first order $(M=1)$ multipactor resonance. Calculations are performed taking, $\omega_{0} b=\pi\left(V_{\text {osc }}+V_{e}\right), \omega_{0} t_{e}=0$, and $V_{e}=0.3 \cdot V_{\text {osc }}$. The dashed black lines on both panels represent the free components of the electron motion. The dotted red line on the bottom panel represents the oscillatory component of the electron velocity, $-V_{\text {osc }} \cos \left(\omega_{0} t\right)(c f$. (4)). 

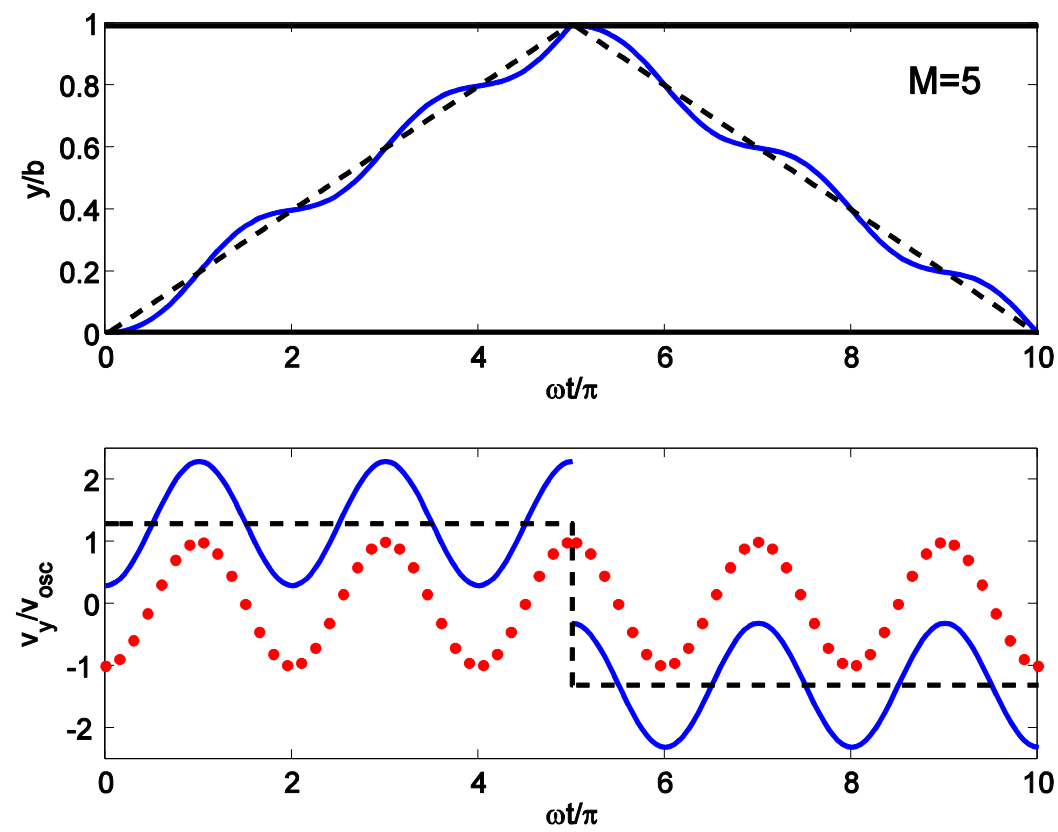

Fig. 2. The same as in Fig. 1, but for the case of fifth order $(M=5)$ multipactor resonance $\left(\omega_{0} b=5 \pi\left(V_{o s c}+V_{e}\right)\right)$.

When considering the multipactor resonance regime, the free component, $V_{f}$, of the electron velocity represents an anti-symmetric step function (Figs. 1-2) with period, $T=2 M \pi / \omega_{0}$, and its Fourier series is calculated to be

$$
V_{f}(t)=u \cdot \sum_{k=2 s+1} \frac{4}{\pi k} \cdot \sin \left[\frac{k \omega_{0}}{M}\left(t-t_{e}\right)\right]
$$

where summation is taken over all odd numbers, $k=2 s+1,\left|\omega_{0} t_{e}\right|<<1$ is the initial rf phase of the resonance regime and $u=V_{o s c} \cos \left(\omega_{0} t_{e}\right)+V_{e} \approx V_{\text {osc }}$. We can then write the current as

$$
J \approx e N V_{o s c}\left[\cos \left(\omega_{0} t\right)-\sum_{k=2 s+1} \frac{4}{\pi k} \cdot \sin \left(\frac{k \omega_{0}}{M} t\right)\right],
$$

The obtained result (5) demonstrate that in the case of the first order $(M=1)$ multipactor resonance, the effective electric current, $J$, contains only integer odd harmonics of the applied electric field. The intensity, $\left|J_{k}\right|^{2}$, of the higher harmonics $(k>1)$ decrease in inverse proportion to $k^{2}$, and the intensity of the third harmonic (the most powerful among the higher harmonics) is about $7 \%$ of the first harmonic intensity $\left(\left|J_{3}\right|^{2} \approx 0.07\left|J_{1}\right|^{2}\right)$. On the other hand, in the case of higher order $(M>1)$ multipactor resonance, the effective electric current, $J$, also contains sub-harmonics of the applied electric field (with frequencies $\omega_{k / M}=k \omega_{0} / M$, and $k<M$ ) [2]. Using Eq. (6) one can find the following relationships for the intensity, $\left|J_{1}\right|^{2},\left|J_{k / M}\right|^{2}$, of different spectral components of the effective current:

$$
\left|J_{1}\right|^{2} \approx(e N)^{2} \cdot V_{o s c}^{2} \cdot\left[1+\left(\frac{4}{\pi M}\right)^{2}\right] \text {, }
$$




$$
\left|\frac{J_{k / M}}{J_{1}}\right|^{2} \approx \frac{(M / k)^{2}}{1+(\pi M / 4)^{2}},
$$

where $k / M \neq 1$. As can be seen from these results the intensity of the main sub-harmonic (at frequency $\omega_{0} / M$ ) can even exceed unity (Figs. 3) [9-11], whereas the relative intensity of the third harmonic $(k=3 M)$ decreases monotonously with an increase in the order of the multipactor $(M)$ resonance $\left(\left|J_{3}\right|^{2} \approx \frac{\left|J_{1}\right|^{2}}{9 \cdot\left(1+(\pi M / 4)^{2}\right)}\right)$. 

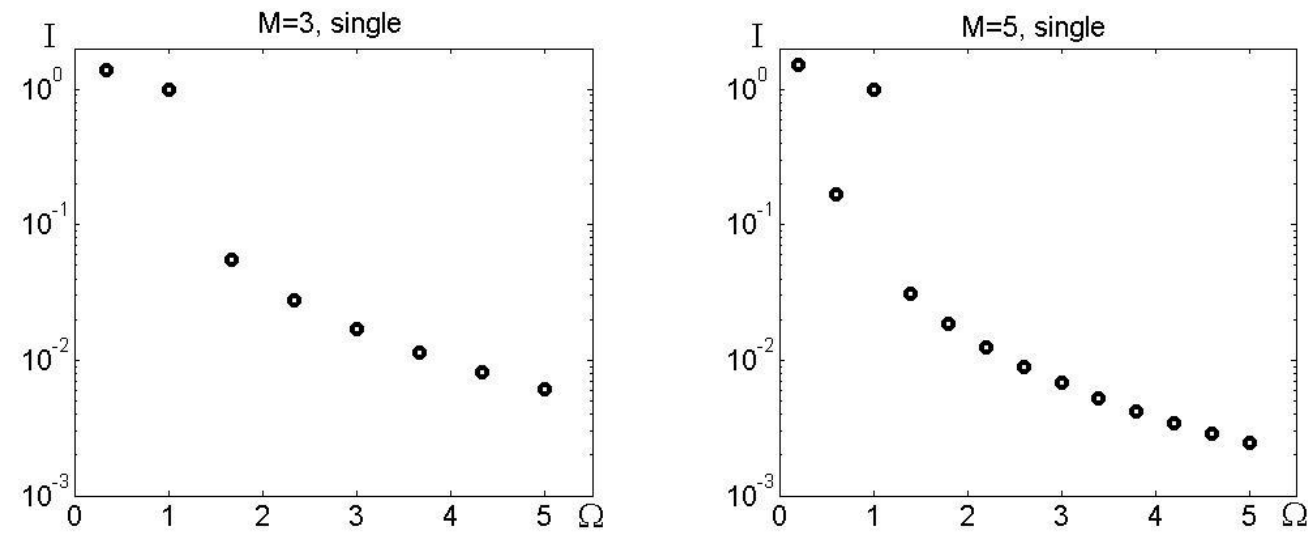

Fig. 3. Normalized spectral intensity of the effective multipactor current, $I=\left|J_{k / M} / J_{1}\right|^{2}$, vs. the relative frequency $\Omega \equiv \omega_{k / M} / \omega_{0}=k / M$. To the left: the case of third order $(M=3)$ multipactor resonance with a single electron sheet. To the right: the case of fifth order $(M=5)$ multipactor resonance with a single electron sheet.
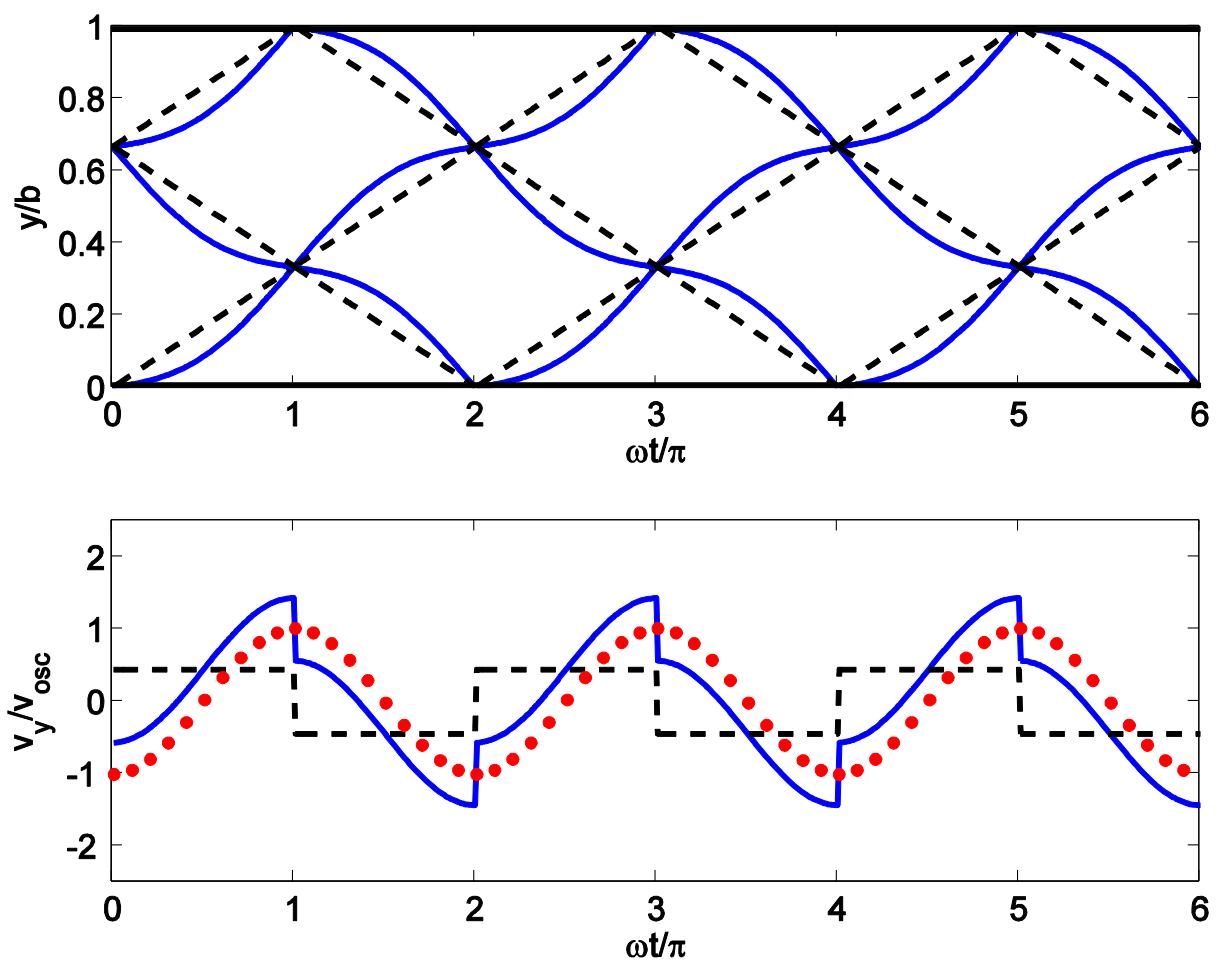

Fig. 4. Electron trajectories and averaged electron velocity (solid blue lines on the top and bottom panel) calculated in the case when the multipactor resonance regime of the third order $(M=3)$ contains three electron sheets with the same number of electrons in each. Calculations are performed using $\omega_{0} b=3 \pi\left(V_{\text {osc }}+V_{e}\right), \omega_{0} t_{e}=0$ and $V_{e}=0.3 \cdot V_{\text {osc }}$. The dashed black lines on the top panel represent the free component of the electron motion in each sheet, whereas in the bottom panel the dashed black line represent the average (over all three sheets) value, $\left\langle V_{f}\right\rangle$, of the free component of the electron velocity. The dotted red line on the bottom panel represents the oscillatory component of the averaged electron velocity, $-V_{\text {osc }} \cos \left(\omega_{0} t\right)(c f .(4))$. 
It should be noted that more than one electron sheet can oscillate simultaneously between the two parallel electrodes in cases involving multipactor resonances of higher order $(M \geq 3)$. These electron sheets are shifted by one or more rf periods with respect to each other (see Fig. 4). When the number of electrons is the same in all electron sheets, the total electric current has the same period as the applied rf field, independently of the resonance order. It is convenient to analyze this current using the concept of an electron velocity which is averaged over all sheets. Specifically, in terms of the average electron velocity, $\langle v\rangle$, the effective electric current can be represented as (cf. Eq. (2))

$$
J \equiv \int\left(\vec{y}_{0} \cdot \vec{j}\right) d y=-e N\langle v\rangle,
$$

where $N$ denotes the total electron number per unit area in all sheets. The oscillatory part of the electron velocity is the same in all sheets since this component does not depend on the emission time (cf. Eq. (4)). Therefore the oscillatory part of the average electron velocity remains the same as in (4). On the other hand, the free component of the electron velocity is quite different in different electron sheets. Simple calculations show that the free component of the average electron velocity represents an anti-symmetric step function with period, $T=2 \pi / \omega_{0}$, and amplitude $\left\langle V_{f}\right\rangle=u / M$. The corresponding Fourier series is calculated to be (cf. Eq. (5))

$$
\left\langle V_{f}(t)\right\rangle=\frac{u}{M} \cdot \sum_{k=2 s+1} \frac{4}{\pi k} \cdot \sin \left[k \omega_{0}\left(t-t_{e}\right)\right],
$$

where summation is taken over all odd numbers, $k=2 s+1,\left|\omega_{0} t_{e}\right|<<1$ is initial rf phase of the resonance regime and $u=V_{\text {osc }} \cos \left(\omega_{0} t_{e}\right)+V_{e} \approx V_{\text {osc }}$. Taking into account the oscillatory part of the average electron velocity one can find the following relations between the harmonic amplitudes, $\left|J_{k}\right|$, of the effective current:

$$
\left|\frac{J_{k}}{J_{1}}\right|^{2} \approx \frac{(1 / k)^{2}}{1+(\pi M / 4)^{2}},
$$

where $k \neq 1$, and $J_{1}$ denotes the spectral amplitude of the first harmonic of the current, which is the same as that given in equation (7). In contrast to multipactor with a single electron sheet, when all possible electron sheets have the same number of electrons the effective electric current, $J$, contains only integer odd harmonics of the applied electric field (see Fig. 5).

The simple models developed above only gives the relative values of the different spectral components of the current. The absolute value of the multipactor electric current cannot be calculated using this approach, since the number, $N$, of multipacting electrons is not determined at the saturation stage. For as was mentioned previously, this number depends on the entire rf circuit. 

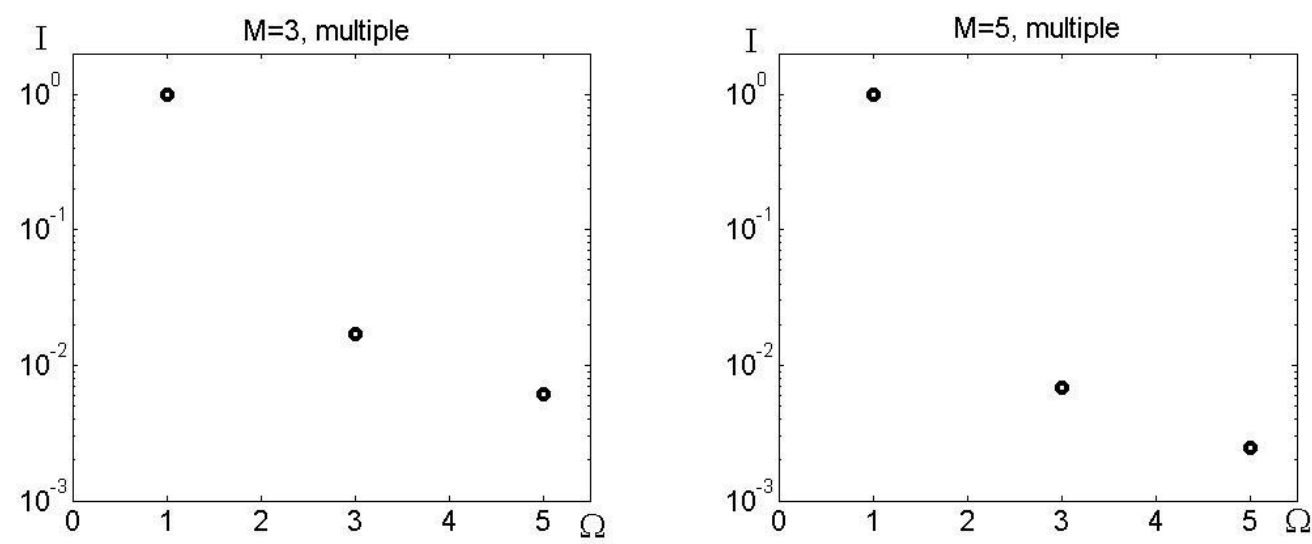

Fig. 5. The same as in Fig. 3 but with three (to the left) and five (to the right) electron sheets having equal amount of electrons.

\section{Numerical simulations of the multipactor current harmonics inside a rectangular waveguide.}

A particular software was developed to simulate the multipactor current in a separate cross-section of a rectangular waveguide. The software is based on an upgraded PIC algorithm [14-16]. When space charge is the multipactor saturation mechanism, the electric field structure will be changed by the presence of the electrons. In our case we assumed that impedance mismatch was the main saturation mechanism, and in this case the electric field strength is reduced but the field structure remains unchanged. This allows us to calculate the electron motion inside the waveguide segment in the undisturbed field of a running electromagnetic wave in the fundamental mode TE10. At the beginning of each simulation run, seed electrons are launched uniformly over several periods of the rf field from the middle of the waveguide wall (the wide wall with the electric field normal to its surface). The seed electrons are assumed to have stochastic emission velocities governed by a Maxwellian probability distribution. The motion of the electrons is governed by both the electric and magnetic fields of the running wave, and each collision of an electron with the metal surface is accompanied by secondary electron emission, being implemented in the simulations as a stochastic process. Its probability distribution is governed by the electron impact energy, and chosen so as to correspond to Vaughan's approximation [17] for the average value, $\sigma\left(W_{i m p}\right)$, of the secondary emission yield:

$$
\begin{aligned}
\sigma & \equiv 0 \text { for } w<0, \\
\sigma=\sigma_{\max } \cdot[w \exp (1-w)]^{s}, s & =0.62 \text { for } 0<w<1, s=0.25 \text { for } w \geq 1,
\end{aligned}
$$

where $\sigma_{\max }=\sigma_{m} \cdot\left(1+\phi^{2} / 4 \pi\right)$ denotes the maximum value of the secondary emission yield including its dependence on the electron impact angle, $\phi$, (with respect to the surface normal). Furthermore $w=\left(W_{i m p}-W_{\min }\right) /\left(W_{\max }-W_{\min }\right)$ where $W_{\text {imp }}$ denotes the electron impact energy, $W_{\max }=W_{m} \cdot\left(1+\phi^{2} / 2 \pi\right)$, and the parameters $\sigma_{\max }, W_{\max }, W_{\min }$ are determined by the material and the surface treatment of the metal plate. The secondary electrons are assumed to start with a stochastic emission velocity governed by the same Maxwellian probability distribution as the seed electrons. The secondary electrons move under the action of the microwave field, until colliding with a wall and giving rise to new secondary electrons, whereupon the process is repeated. 


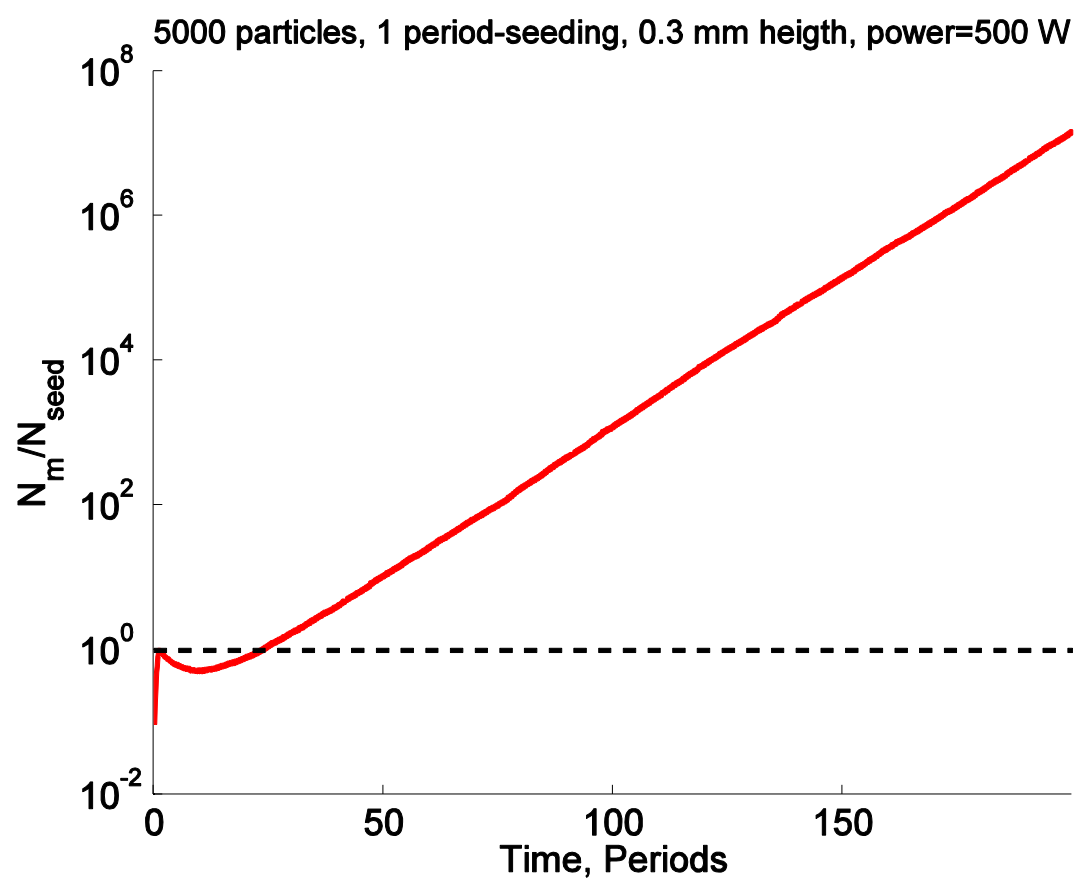

Fig. 6. The relative number, $N_{m} / N_{\text {seed }}$, of multipacting electrons vs. time (in rf periods). Calculations are done taking 2500 seed electrons launched during one rf period for an aluminum waveguide with width, $a=58.17 \mathrm{~mm}$, height, $b=0.3 \mathrm{~mm}$, at rffrequency, $f=4.2 \mathrm{GHz}$, and input power,

$$
P=500 \mathrm{~W} \text {. }
$$

The calculation data is stored, which makes it possible to study the evolution of both the electron number and the electron current as a function of time. The multipactor threshold is found by studying the evolution of the electron number. Within the approach used in this study, the multipactor avalanche does not saturate and the electron number grows during the whole simulation time, provided the input rf power exceeds the threshold value (Fig. 6). The initial dip in the electron population in Fig. 6 is caused by the fact that seed electrons are launched uniformly over the field period for several periods. The electrons that are launched out of resonance will quickly disappear, whereas those launched at the proper resonant phase will lead to the eventual electron growth. However, the simulations show that after sufficiently long time, when a multipactor avalanche is completely established, the specific electric current (the ratio of the multipactor current to the number of multipacting electrons) becomes a quasi-periodic function of time (see Fig. 7). Based on a previous study of multipactor inside a rectangular waveguide [18], it is possible to state that in the case when the waveguide height is much less than the electromagnetic wavelength, the multipactor avalanche grows independently in each waveguide cross-section and its growth rate is determined by the local amplitude of the rf electric field. Therefore in this case the simulation results which are obtained for a running electromagnetic wave can also be applied to describe multipactor in standing and mixed waves. Correspondingly it seems reasonable to assume that the specific current, which is established in the case of a growing multipactor avalanche, is the same as that in the case of multipactor saturation provided the latter is caused by impedance mismatch.

All simulations were carried out using the parameters $\sigma_{m}, W_{\min }, W_{m}$ so as to fit the secondary emission of oxidized aluminum given by CNES (maximum $\sigma=2.93$, first cross-over point $W_{1}=23 \mathrm{eV}$, and due to partial electron reflection it was assumed that $\sigma \rightarrow 0.5$ when electron impact energy goes to zero). The frequency was taken to be $f=4.2 \mathrm{GHz}$, and the waveguide width $a=58.17 \mathrm{~mm}$. The waveguide height, $b$, was varied to check the dependence of the current harmonics on the order of the multipactor resonance. For each value of the waveguide height, a series 
of simulations was completed with different input power which makes it possible not only to find the multipactor threshold, but also to study how sensitive the specific multipactor current is to an excess of power over the multipactor threshold.

The first simulation series was completed taking the waveguide height to be $b=0.3 \mathrm{~mm}$, which made it possible to study the first order multipactor resonance $(M=1)$. In this case the multipactor threshold (in terms of input power) was found to be $P_{t h} \approx 478 \mathrm{~W}$. For any power exceeding the multipactor threshold, the software calculates the spatiotemporal evolution of the specific current density related to the waveguide cross-section under consideration:

$$
j_{s p}(x, y, t)=\left(\vec{y}_{0} \cdot \vec{j}\right) \frac{\Delta z}{N_{m}},
$$

where $\Delta z$ denotes the effective thickness of the multipactor current slab associated with the considered waveguide cross-section, and $N_{m}$ is the instantaneous number of multipacting electrons. In the simulations, the electrons $N_{m}$ move inside the simulation volume, $a b \Delta z$, where the field is taken as homogeneous in the $\mathrm{z}$-direction. This approach is valid provided $\beta \Delta z<<1$, where $\beta$ is the propagation constant in the waveguide.

The electrons move up and down throughout the full width of the waveguide, and their ability to generate different EM modes able to propagate through the waveguide depend on their spatial distribution along the $\mathrm{x}$-axis. The $\mathrm{TE}_{10}$ mode is generated by a current on the form $\sin (\pi x / a)$, the $\mathrm{TE}_{30}$ mode by a current on the form $\sin (3 \pi x / a)$ etc. Due to the low height of the waveguide, there will be no propagating modes with a nonzero second index, e.g. $\mathrm{TE}_{11}$. The different modal components of the current, $I_{m}(t)$, can be found from the specific electric current using

$$
I_{m}(t)=\frac{1}{b} \int j_{s p}(x, y, t) \cdot \sin \left(\pi \frac{m x}{a}\right) d x d y,
$$

where integration is taken over the waveguide cross-section. The distribution of electrons along the $\mathrm{x}$ axis will be determined mainly by the power and the resonance criteria. For only in regions where the electric field, the gap width and the frequency fulfills certain resonance criteria will an electron avalanche be able to develop. Consequently the rf power will have a great influence on which modes are generated and propagated due to multipactor. Fig. 7 presents the first modal component $I_{1}(t)$ of the specific multipactor current in the first simulation series. 

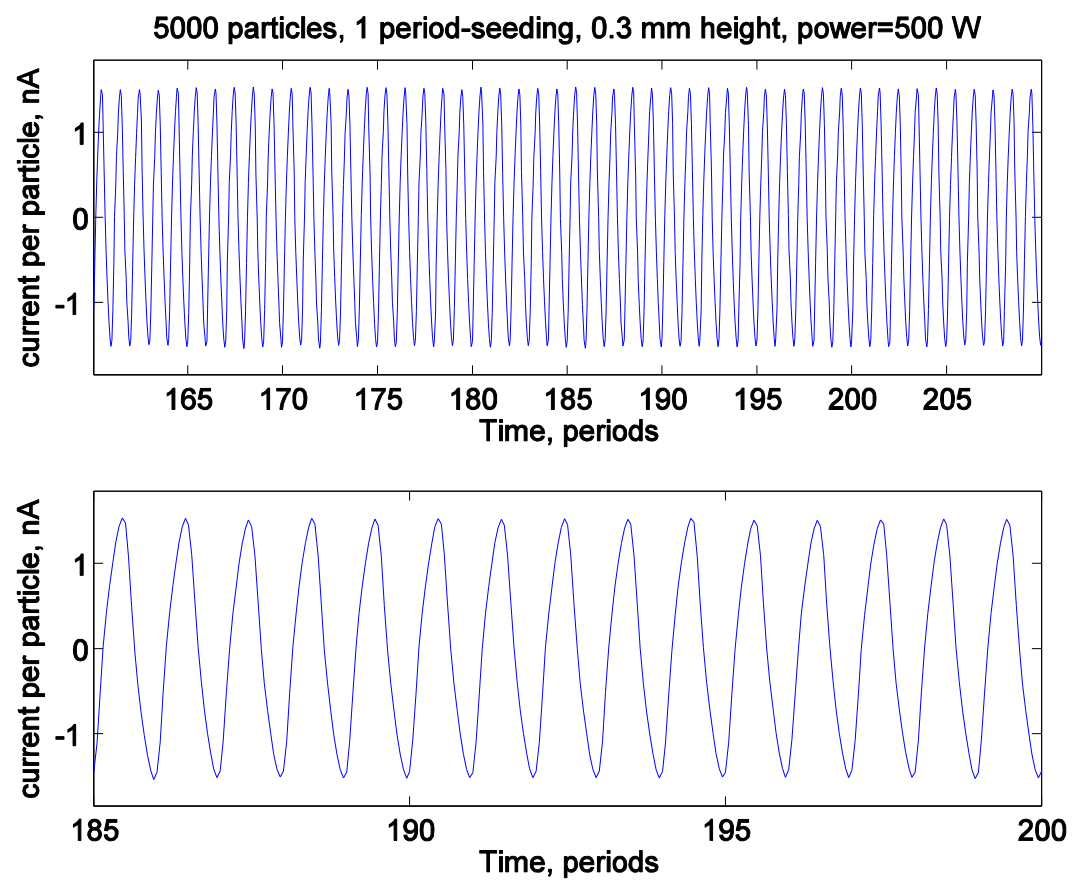

Fig. 7. Established evolution of the specific electric current (the first modal component $I_{1}(t)$ of the multipactor current per electron). The bottom panel presents the same curve as the top panel but with higher resolution. The simulation parameters are the same as in Fig. 6.

Each of the modal components will be composed of a range of frequencies, including both the harmonics and the noise caused by the stochastic emission velocity. One can find the specific amplitude spectrum of the modal components using

$$
I_{m}(\omega)=\left|\frac{1}{T} \int_{t_{0}}^{t_{0}+T} I_{m}(t) \cdot \exp (-i \omega t) d t\right|,
$$

where the integration starts from $t_{0}=150 \mathrm{rf}$ periods and is taken over $T=50 \mathrm{rf}$ periods. The function $I_{m}(\omega)$ determines the spectrum of the radiated electromagnetic power for the mode $\mathrm{TE}_{\mathrm{m} 0}$. The specific amplitude spectrums for the three first modes (TE10, TE30, TE50) can be seen in Fig.8 vs. the normalized frequency $\omega / \omega_{0}$ (where $\omega_{0}=2 \pi \cdot f$ is the angular frequency of the input rf field). Here the simulation results are presented for an input power which is slightly above the threshold value when the multipactor current is localized close to the middle plane of the waveguide and the modal currents, $I_{1}(t), I_{3}(t), I_{5}(t)$ almost coincide with each other, as can be seen by the close overlapping of the blue, green and red lines in Fig. 8. In complete agreement with the theoretical predictions, the spectrum (shown in Fig. 8) contains well pronounced peaks at odd harmonics of the input frequency and background noise with a level that decreases with increasing number of macroparticles used in the simulations. It should be noted that the ratio between the heights of the peaks of different harmonics is close to that given by equation (8) for the first order resonance ( $M=1)$. 

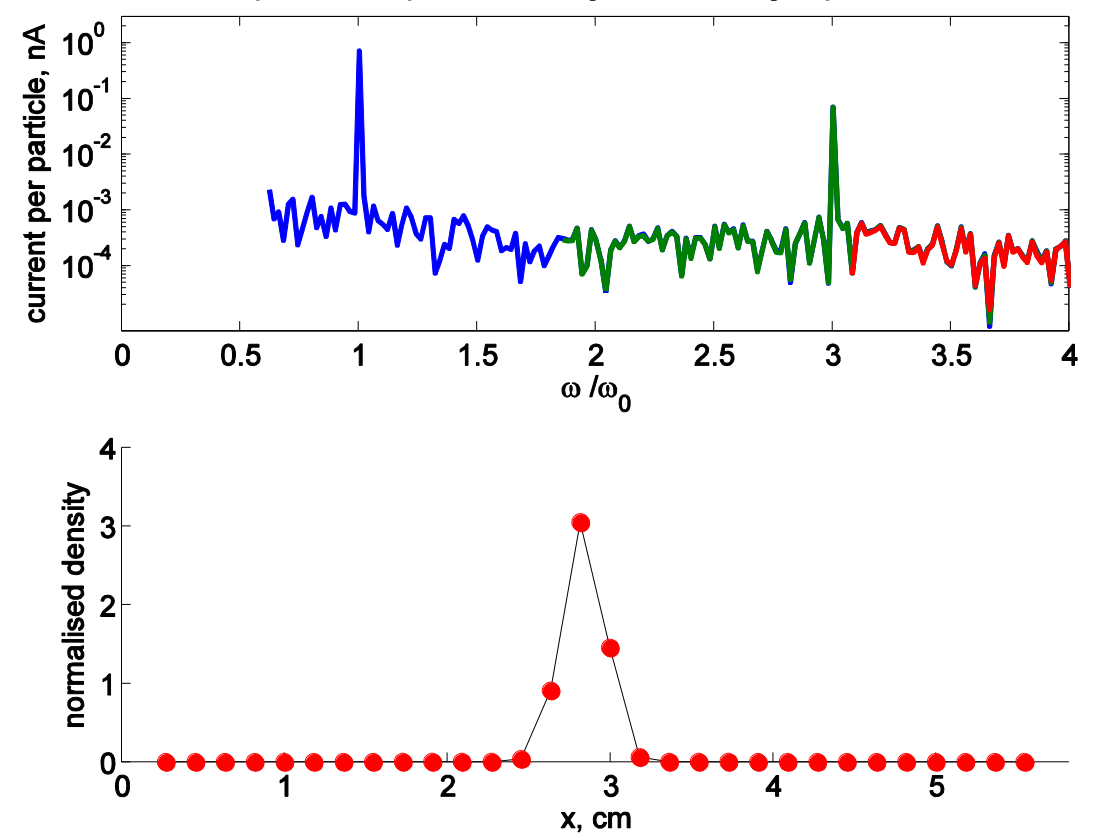

Fig. 8. Top panel: the amplitude spectrum of the modal multipactor current per one electron. Blue, green, and red lines represent $I_{1}(\omega), I_{3}(\omega)$, and $I_{5}(\omega)$ respectively. Each line is shown only for the frequency interval where the corresponding mode is propagating. Bottom panel: the spatial distribution of the normalized electron density (obtained after $110 \mathrm{rf}$ periods) along the wide wall of the waveguide. The multipacting electrons are well localized in the middle of the waveguide.

Simulations are completed for the waveguide height, $b=0.3 \mathrm{~mm}$, at the input rf power $500 \mathrm{~W}$, with 2500 seed electrons launched during one rf period. The online version of this figure is in color.

A number of simulations were carried out in order to understand how sensitive the amplitude spectrum of the specific multipactor is to the applied rf power. The results of those simulations demonstrate that an increase in the rf power is accompanied by an increase in the amplitude of all the harmonics of the specific current (see Fig. 9). The rate of increase in the amplitudes of the higher harmonics was found to be faster than that of the first harmonic amplitude. At the same time, the difference between the first and the third modal amplitudes is almost negligible within a relatively wide interval of power. 

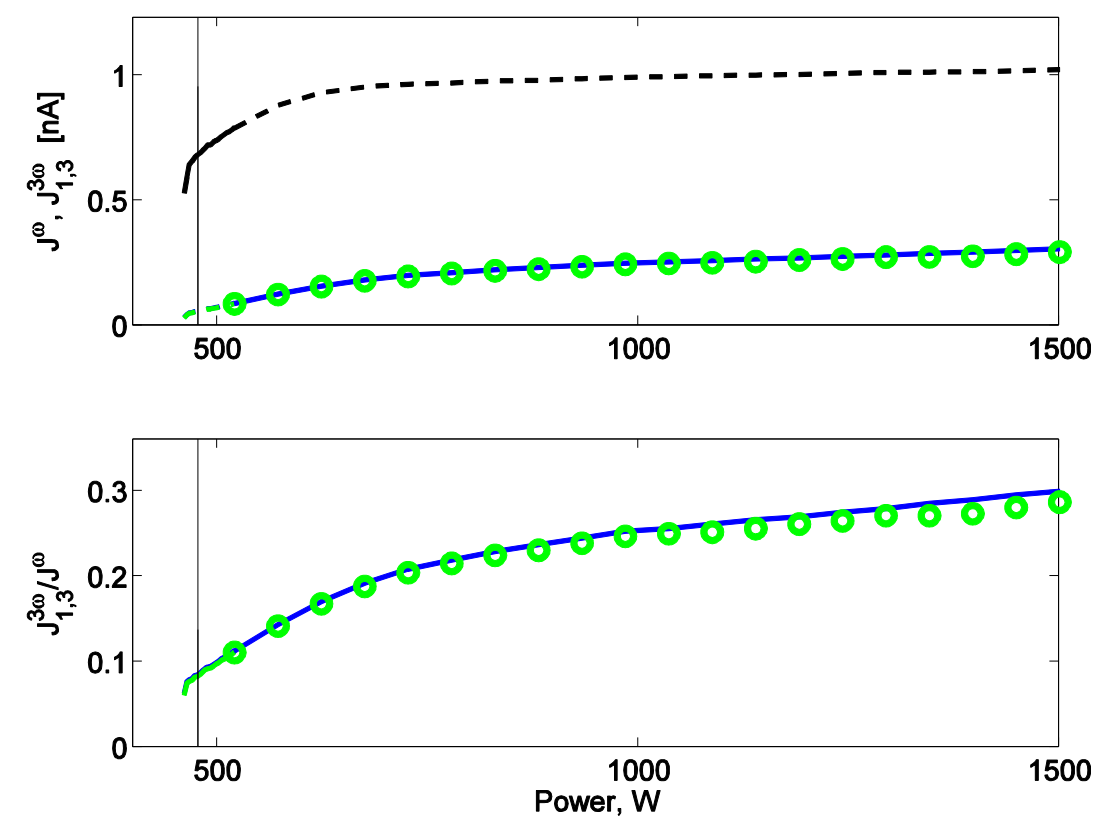

Fig. 9. Top panel: amplitudes of the modal harmonics of the specific current vs. the rf power. Black dashed line corresponds to the first harmonic of the first mode, $J^{\omega} \equiv I_{1}\left(\omega_{0}\right)$, blue solid line to the third harmonic of the first mode, $J_{1}^{3 \omega} \equiv I_{1}(3 \omega)$, and green circles to the third harmonic of the third mode, $J_{3}^{3 \omega} \equiv I_{3}(3 \omega)$. Bottom panel: the ratio $J_{1}^{3 \omega} / J^{\omega}$ (blue solid line) and $J_{3}^{3 \omega} / J^{\omega}$ (green circles) vs. power. Vertical lines indicate the multipactor threshold power on both panels. Simulations are completed for the waveguide height $b=0.3 \mathrm{~mm}$, taking 2500 seed electrons launched during one $\mathrm{rf}$ period.

In the second series, the simulations were made for a waveguide with a height, $b=0.9 \mathrm{~mm}$ which makes it possible to study the multipactor resonance of the third order $(M=3)$. In this case the multipactor threshold (in terms of input power) is found to be $P_{t h} \cong 2100 \mathrm{~W}$. Fig. 10 shows that an exponential increase in the electron number is accompanied by the establishment of a quasi-periodic current per electron. In the case of third order multipactor resonance, one can observe some fluctuations in the established evolution of the specific current which are more pronounced as compared to the case of the first order resonance (cf. Fig. 7 and Fig. 10). The specific amplitude spectra for the three first modes $\left(\mathrm{TE}_{10}, \mathrm{TE}_{30}, \mathrm{TE}_{50}\right)$ can be seen in Fig.11. Here the simulation results are presented for two different values of input power. When the power is slightly above the threshold value, the multipactor current is localized close to the middle plane of the waveguide and the modal currents, $I_{1}(t), I_{3}(t), I_{5}(t)$ almost coincide with each other, similarly to the case of the first order multipactor resonance. Some similarity with the first order resonance can also be found by looking at the amplitude spectra of modal currents, which clearly demonstrates pronounced peaks at odd harmonics of the input frequency, whereas the sub-harmonic peaks (at the frequencies $5 \omega_{0} / 3$ and $7 \omega_{0} / 3$ which are indicated in Fig. 3) are not well pronounced against the background noise. In this sense, at a power which is close to the multipactor breakdown threshold, the spectrum in Fig. 11 looks similar to that predicted theoretically for third order multipactor resonance with three electron sheets oscillating simultaneously between the electrodes (cf. Fig. 5). It should be noted that the ratio between the heights of the peaks of different harmonics is also close to that given by equation (11) for third order resonance ( $M=3$ ) with three electron sheets. 

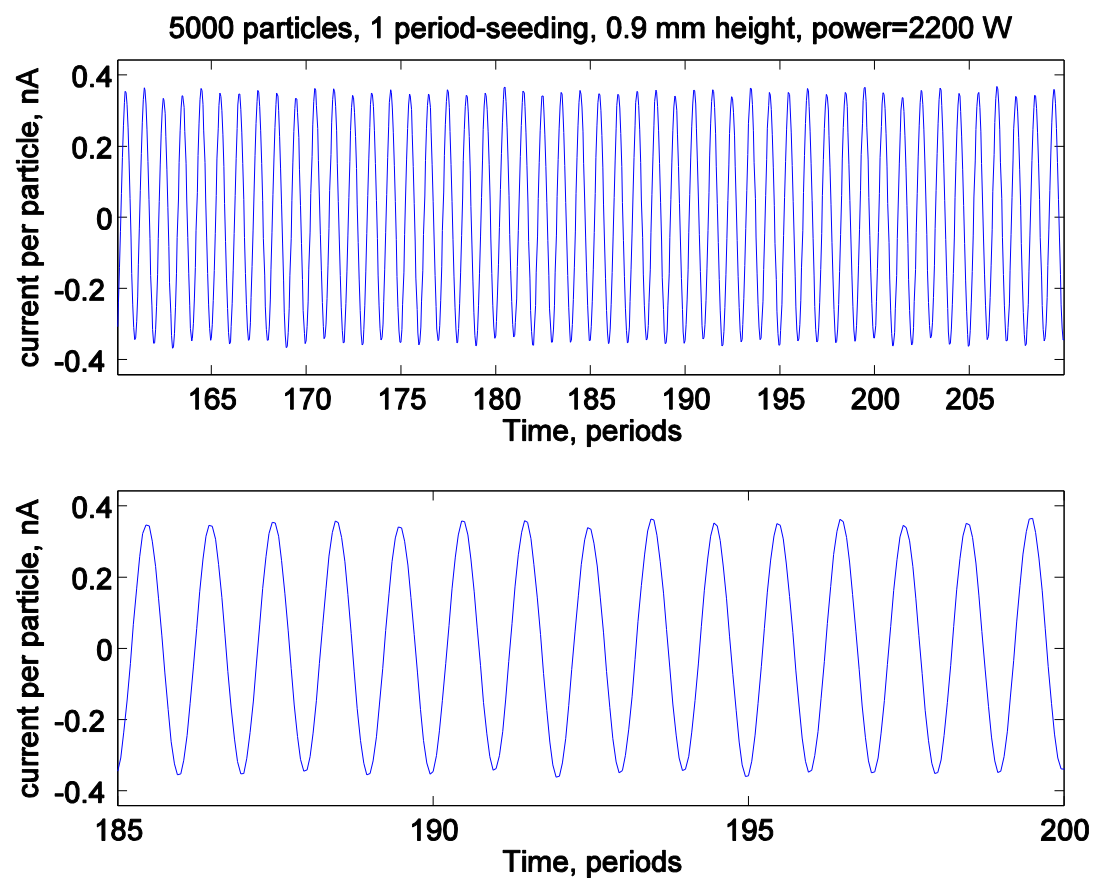

Fig. 10. Established evolution of the specific electric current (the first modal component $I_{1}(t)$ of the multipactor current per electron). The bottom panel presents the same curve as the top panel but with higher resolution. Calculations are done taking 2500 seed electrons launched during one rf period for an aluminum waveguide with width, $a=58.17 \mathrm{~mm}$, height, $b=0.9 \mathrm{~mm}$, at rf frequency, $f=4.2$

GHz and input power, $P=2200 \mathrm{~W}$.

However, a more detailed study of this spectrum (taking into account the non-propagating modes) revealed the existence of a sub-harmonic current peak. In contrast to the theoretical predictions for multipactor with a single electron sheet, the height of the revealed sub-harmonic peak is less than the height of the first harmonic peak, which makes it possible to conclude that in the simulations there were three electron sheets but with different number of electrons in each. Whether the peak belonging to the non-propagating mode will lead to any noise in a realistic system depends on the length of the waveguide and if outside the waveguide there are propagating modes at this frequency. Additional simulations demonstrated that in the case of third order multipactor resonance the multipactor current is more sensitive to the level of input power. As can be seen from the bottom panel of Fig. 11, the subharmonic peaks become much more pronounced, along with the difference between different modal components. 


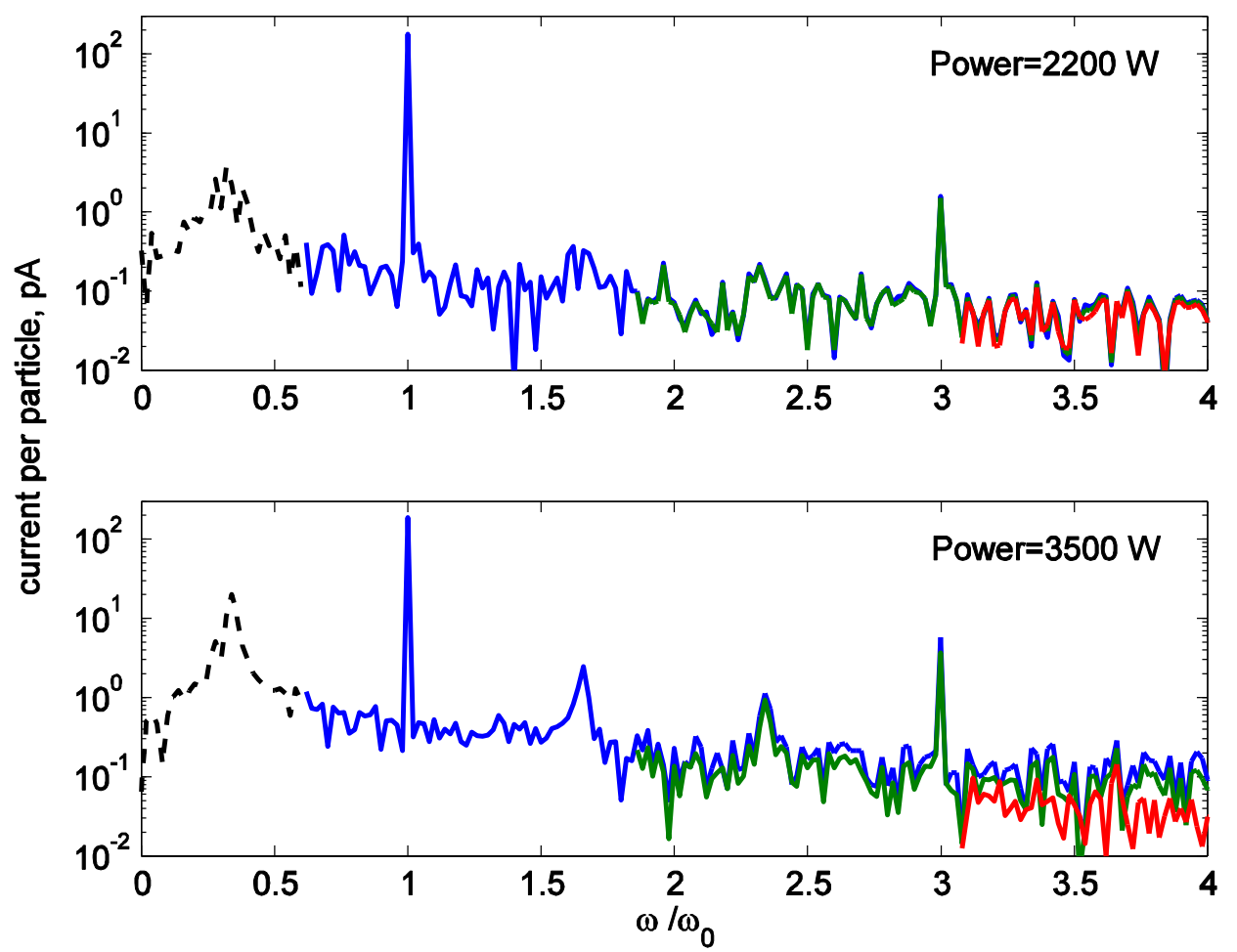

Fig. 11. The amplitude spectrum of the modal multipactor current per electron calculated for $2200 \mathrm{~W}$ (top panel) and $3500 \mathrm{~W}$ (bottom panel). Other simulation parameters are the same as in Fig. 10. The solid color lines (blue, green, and red) represent the spectra of different modal components $\left(I_{1}(\omega)\right.$, $I_{3}(\omega)$, and $I_{5}(\omega)$ ). Black dashed line represents $I_{1}(\omega)$ in the frequency range where the first mode does not propagate. The online version of this figure is in color.

In the third series of multipactor simulations the height of the waveguide was increased further. The simulation results do not reveal any new qualitative feature in the spectra of modal currents. It was found in these simulations that the frequency separation between different spectral peaks decreases monotonously with an increase in the waveguide height, in complete agreement with the theoretical predictions (cf. two panels of the Fig. 3). Specifically, inside a waveguide with height $b=4.5 \mathrm{~mm}$, at a power level slightly above the multipactor threshold, no spectral peaks were found (with exception of the first harmonic peak) that could be clearly distinguished against the background noise (see top panel in Fig. 12). An increase of the power level inside this waveguide makes the third harmonic peak more pronounced, while all the other spectral peaks remain indistinguishable against the background noise (see bottom panel on Fig. 12). 

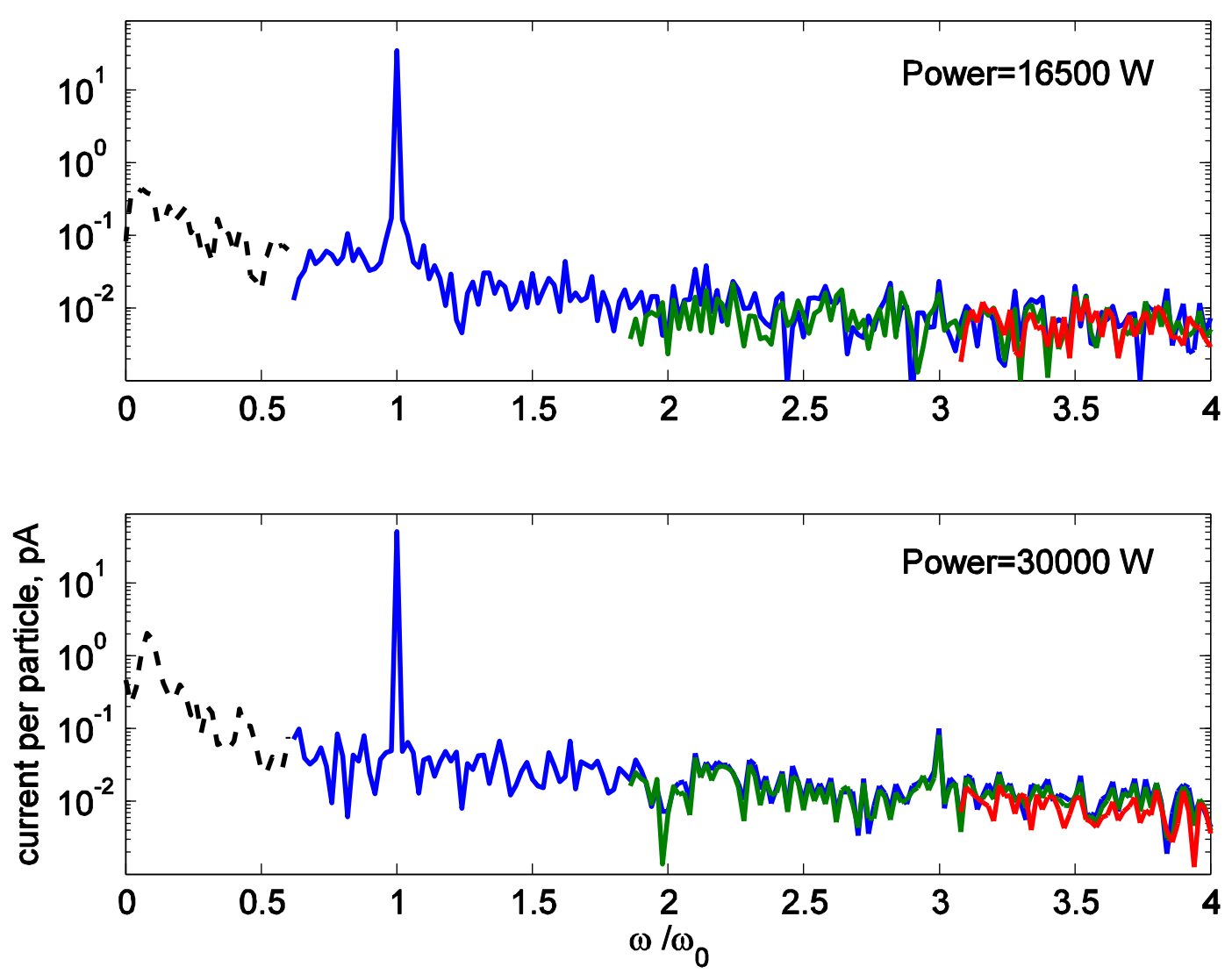

Fig. 12. The amplitude spectrum of the modal multipactor current per electron calculated for the power $16500 \mathrm{~W}$ (top panel) which is close to the multipactor threshold ( $P_{\text {th }} \cong 16000 \mathrm{~W}$ ), and for

higher power $30000 \mathrm{~W}$ (bottom panel). The waveguide height is $b=4.5 \mathrm{~mm}$, which makes it possible to reach multipactor resonance of the 15-th order. The simulations were completed taking 2500 seed electrons launched during one rf period. The solid color lines (blue, green, and red) represents spectra of different modal components $\left(I_{1}(\omega), I_{3}(\omega)\right.$, and $I_{5}(\omega)$ ). Black dashed line represents $I_{1}(\omega)$ in the frequency range where the first mode does not propagate. The online version of this figure is in color.

To check the sensitivity of the background current noise to the number of macroparticles used by the software, the multipactor simulations were repeated for a waveguide with height $b=0.9 \mathrm{~mm}$ using a greater number of macro-particles (20000 instead of 5000). The simulation results (Fig. 13) clearly demonstrate a reduction both of the background noise and of the sub-harmonic peaks with an increase in the number of macro-particles, which is associated with a reduction in the stochastic fluctuations. One more numerical test was performed for this waveguide launching seed electrons during three rf periods. In this case it seemed more probable to get the multipactor discharge with multiple electron sheets in simulations. In complete agreement with this assumption the simulation results (Fig. 14) demonstrate a significant reduction of background noise and sub-harmonic peaks. 


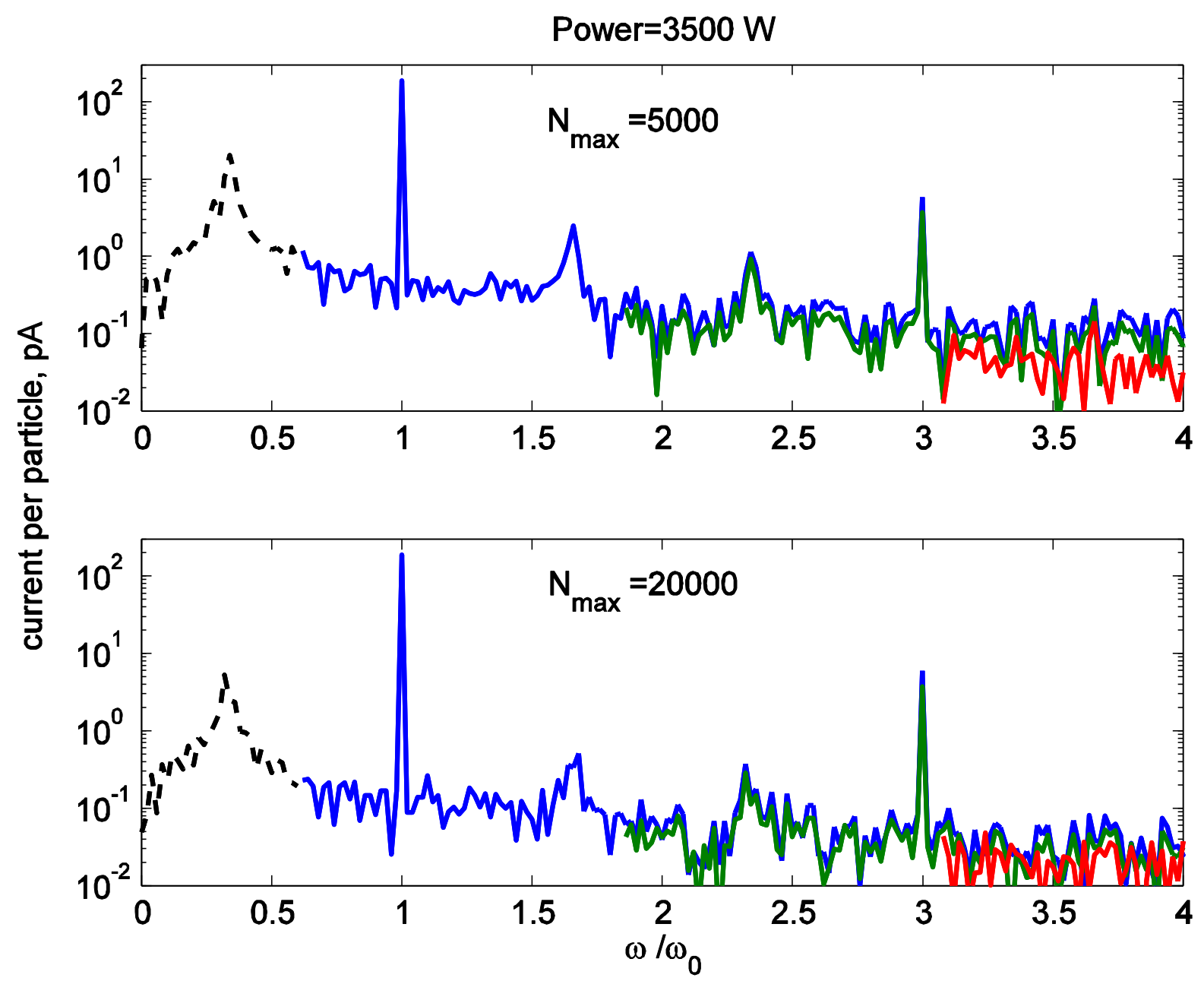

Fig. 13. Top panel represents the same results as in the bottom panel of Fig. 11. Bottom panel present results of similar simulations using a larger number $N_{\max }=20000$ of macro-particles. The online version of this figure is in color. 


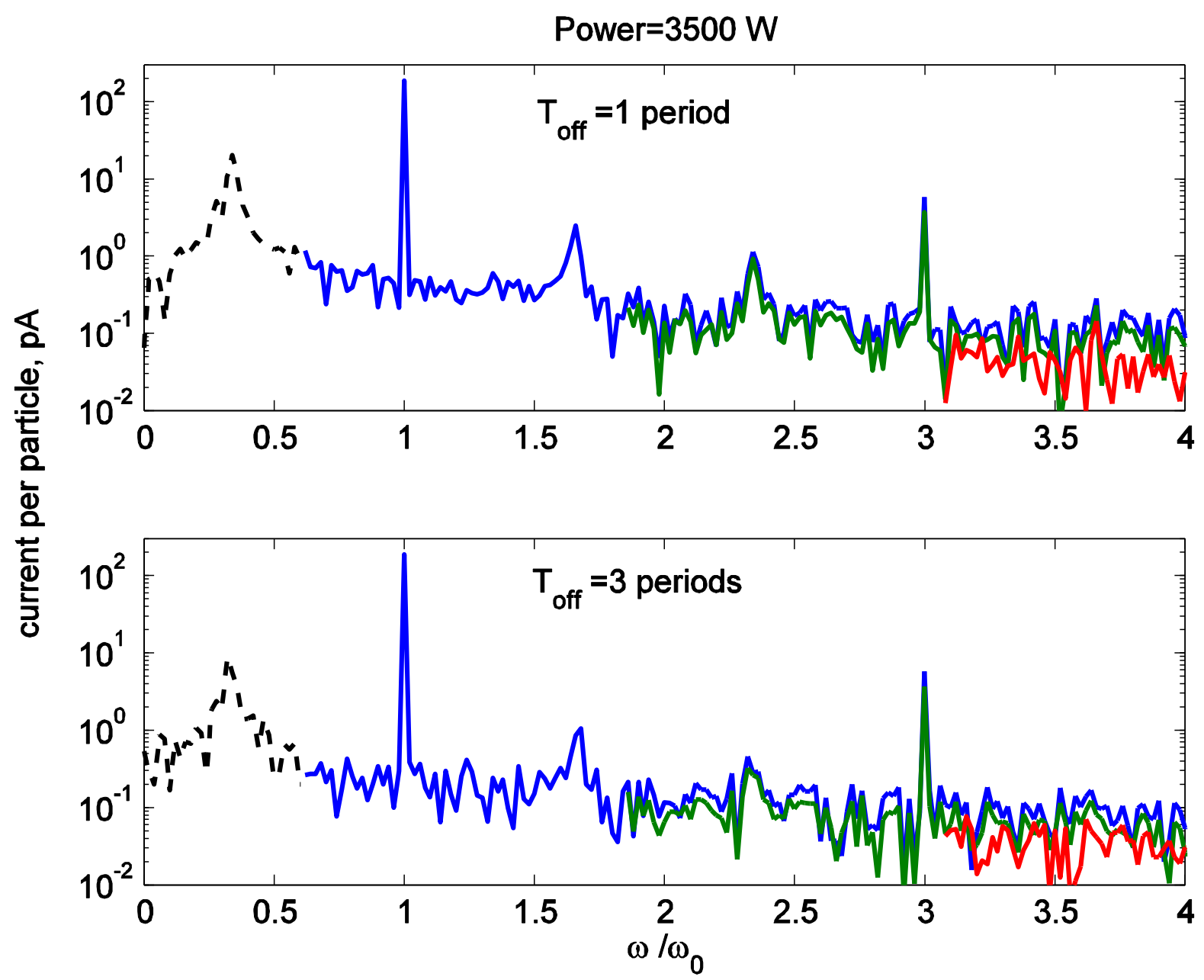

Fig. 14. Top panel represents the same results as in the bottom panel of Fig. 11. Bottom panel present results of similar simulations using a larger temporal interval ( $3 \mathrm{rf}$ periods) for the launch of seed electrons. The online version of this figure is in color.

\section{Conclusions}

A study of the multipactor noise has been undertaken using both analytical theory and numerical simulations. The study is based on the concept of an effective electric current, caused by the multipacting electrons, which determines the output electromagnetic noise from a device. Simple analytical estimates are obtained for the amplitude spectrum of the effective electric current when applying the resonance theory to a multipactor discharge between two plane-parallel electrodes exposed to a monochromatic rf voltage. It is shown that in the case of first order multipactor resonance the effective current contains only odd integer harmonics of the applied frequency, whereas in the case of higher order multipactor resonance the effective current can also contain sub-harmonics of the applied frequency. These predictions were confirmed by numerical simulations of multipactor inside a rectangular waveguide. In the simulations, a continuous spectrum of background multipactor noise was also found. And the mechanism behind the generation of this noise was determined to be the stochastic fluctuations of the electron emission velocity. It was demonstrated that an increase in the waveguide height is accompanied by a considerable reduction in the height of the current spectral peaks at harmonics of applied frequency. And the reduction became sufficiently great to make the peaks indistinguishable against the background noise when the waveguide height exceeded a value sufficiently large to allow multipactor resonance of the $15^{\text {th }}$ order. 


\section{References}

[1] R. Udiljak, D. Anderson, P. Ingvarson, U. Jordan, U. Jostell, L. Lapierre, G. Li, M. Lisak, J. Puech, and J. Sombrin, "New Method for Detection of Multipaction", IEEE Trans. Plasma Sci., 31, 3, pp. 396-404 (2003).

[2] A. J. Marrison, R. May, J. D. Sanders, A. D. Dyne, A. D. Rawlins, and J. Petit, A Study of Multipaction in Multicarrier RF Components, AEA Technology for ESTEC, AEA Ref. No. AEA/TYKB/31761/01/RP/05 Issue 1 (Culham, UK, 1997).

[3] M. Buyanova, V. E. Semenov, D. Anderson, M. Lisak, and J. Puech, "Influence of secondary emission yield on the saturation stage of multipactor discharges between two parallel metal plates", Phys. Plasmas, 17, 043504 (2010).

[4] R. Kishek, and Y.Y.Lau, "Interaction of Multipactor Discharge and rf Circuit", Phys. Rev. Letters, 75, 6, pp. 1218-1221 (1995).

[5] R. A. Kishek, and Y. Y. Lau, "A novel phase focusing mechanism in multipactor discharge", Phys. Plasmas, 3, 5, 1481-1483 (1996).

[6] S. Riyopoulos, "Multipactor saturation due to space-charge-induced debunching", Phys. Plasmas, 4, 5, 1448-1462 (1997).

[7] E. Sorolla, and M. Matter, "Multipactor saturation in parallel-plate waveguides", Phys. Plasmas, 19, 7, 072304 (2012).

[8] R. A. Kishek, Y. Y. Lau, and D. Chernin, "Steady state multipactor and dependence on material properties", Phys. Plasmas, 4, 3, pp. 863-872 (1997).

[9] S. Anza, C. Vicente, D. Raboso, J. Gil, B. Gimeno, and V. E. Boria, "Enhanced prediction of multipaction breakdown in passive waveguide components including space charge effects", Microwave Symposium Digest, 2008 IEEE MTT-S, pp. 1095 - 1098.

[10] B. Gimeno, E. Sorolla, S. Anza, C. Vicente, J. Gil, A. M. Pérez, V. E. Boria, F. J. Pérez-Soler, F. Quesada, A. Álvarez, and D. Raboso, "Multipactor radiation analysis within a waveguide region based on a frequency-domain representation of the dynamics of charged particles", Phys. Rev. E, 79, 4, 046604 (2009).

[11] M. Jiménez, B. Gimeno, C. Miquel-Espanya, D. Raboso, S. Anza, C. Vicente, J. Gil, F. Quesada, A. Álvarez, M. Taroncher, M. Reglero, and V. E. Boria, "Analysis of the electromagnetic radiation generated by a multipactor discharge occurring within a microwave passive component", J. Phys. D: Appl. Phys., 43, 395501 (2010)

[12] M. Jimenez, B. G. Martinez, D. Raboso, S. Anza, A. Alvarez, F. Quesada, V. E. Boria, C. Vicente, and J. Gil, "Multimodal Characterization of the Multipactor Effect in Microwave Waveguide Components", IEEE Microwave and Wireless Components Letters, 22, 2, 61-63 (2012).

[13] E. Sorolla, S. Anza, B. Gimeno, A. M. Pérez, C. Vicente, J. Gil, F. J. Pérez-Soler, F. D. Quesada, A. Álvarez, and V. E. Boria, "An Analytical Model to evaluate the Radiated Power Spectrum of a Multipactor Discharge in a Parallel-Plate Region", IEEE Trans. Electron Dev., 55, 8, pp. 2252-2258 (2008). 
[14] V. Semenov, E. Rakova, N. Zharova, D. Anderson, M. Lisak, and J. Puech, "Simulations of the Multipactor Effect in Hollow Waveguides with Wedge-Shaped Cross Section", IEEE Trans. Plasma Sci., 36, 2, pp. 488-493 (2008).

[15] I. A. Kossyi, G. S. Luk'yanchikov, V. E. Semenov, N. A. Zharova, D. Anderson, M. Lisak, and J. Puech, "Experimental and Numerical Investigation of Multipactor Discharges in a Coaxial Waveguide", J. Appl. Phys. D: Applied Physics, 43, 345206 (2010).

[16] V. E. Semenov, N. A. Zharova, D. Anderson, M. Lisak, and J. Puech, "Simulations of Multipactor in Circular Waveguides", Phys. Plasmas, 17, 123503 (2010).

[17] R. M. Vaughan, “Secondary Emission Formulas”, IEEE Trans. Electron Dev., 40, 4, pp. 830-830 (1993).

[18] V. E. Semenov, E. I. Rakova, D. Anderson, M. Lisak, and J. Puech, "Multipactor in rectangular waveguides", Phys. Plasmas, 14, 033501 (2007). 